Research Article

\title{
Application of a Recurrent Neural Network and Simplified Semianalytical Method for Continuous Strain Histories Estimation
}

\author{
Huan Luo $\mathbb{D}^{1},{ }^{1}$ Miaohua Huang $\mathbb{D}^{2},{ }^{2}$ and Wei Xiong $\mathbb{D}^{1}$ \\ ${ }^{1}$ GAC Automotive Research \& Development Center, Guangzhou 511434, China \\ ${ }^{2}$ Hubei Key Laboratory of Advanced Technology for Automotive Components, Wuhan University of Technology, \\ Wuhan 430070, China \\ Correspondence should be addressed to Huan Luo; luohuan616@163.com
}

Received 29 December 2018; Revised 28 March 2019; Accepted 31 March 2019; Published 16 June 2019

Academic Editor: Mario Terzo

Copyright $\odot 2019$ Huan Luo et al. This is an open access article distributed under the Creative Commons Attribution License, which permits unrestricted use, distribution, and reproduction in any medium, provided the original work is properly cited.

The durability and reliability of structural components are usually assessed based on fatigue loading under operating conditions. To obtain accurate fatigue loading in the form of continuous strain histories, a novel approach is proposed based on the combination of a recurrent neural network and simplified semianalytical method. The recurrent neural network named nonlinear autoregressive model with exogenous inputs (NLARX) is applied to determine the relationship between external loads and corresponding fatigue loading. Owing to the generalization ability of NLARX, semianalytical method, which is used to obtain sample database for NLARX model training and testing, is implemented with simplified multibody model. Durability tests of a torsion beam rear suspension are introduced to demonstrate the effectiveness of the proposed approach. The experimental results show that our proposed approach is able to achieve better estimation results, when compared with the conventional semianalytical method.

\section{Introduction}

Automotive load-bearing components usually subject to various random loads and are vulnerable to fatigue damage. Thence, accurately predicting fatigue life at durability design stage is critical for the reliability of these automotive components. According to the theory used for fatigue life prediction and fatigue damage analysis [1-3], fatigue loading in the form of stress-time or strain-time histories is the key factor affecting outcome. Therefore, accurately estimating fatigue loading is of great importance in the process of durability design.

Semianalytical method, which is a mix of simulation analyses and automotive durability tests, is a feasible solution due to its low cost and high efficiency [4]. As shown in Figure 1(a), the basic framework behind semianalytical method consists of four major parts: durability tests on proving ground, multibody dynamic simulation, finite element transient analysis, and structural damage calculation. In this method, fatigue loading is the output result of finite element transient analysis. Though widely applied in automotive field, the conventional semianalytical method still cannot lead to satisfactory results in many cases, due to the existence of modelling error and calculator error in the process of multibody dynamic simulation and finite element transient analysis [5-8]. As a result, obtaining accurate fatigue loading remains challenging and has attracted considerable research efforts.

In order to improve the calculation accuracy of fatigue loading, many improved methods have been developed. In [9-11], the researchers build more accurate full-vehicle model for multibody dynamic simulation. As an example, the full-vehicle model with new road tire model can lead to more accurate results [9]. In [12-14], more appropriate form of boundary constraint is designed for multibody dynamic simulation. Liu has demonstrated that accurate external 


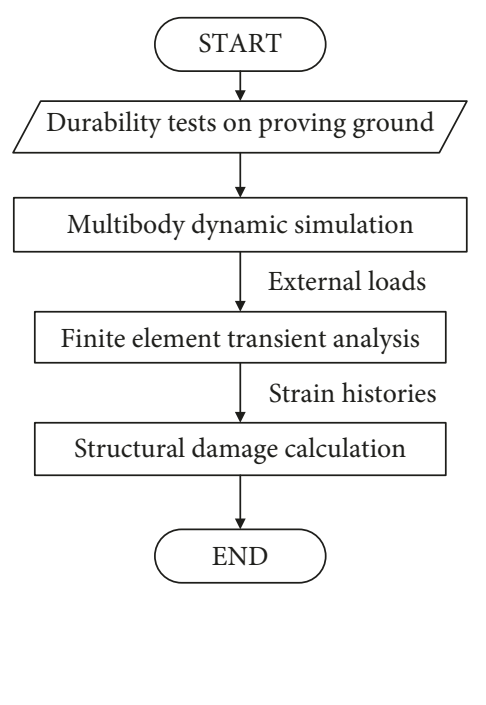

(a)

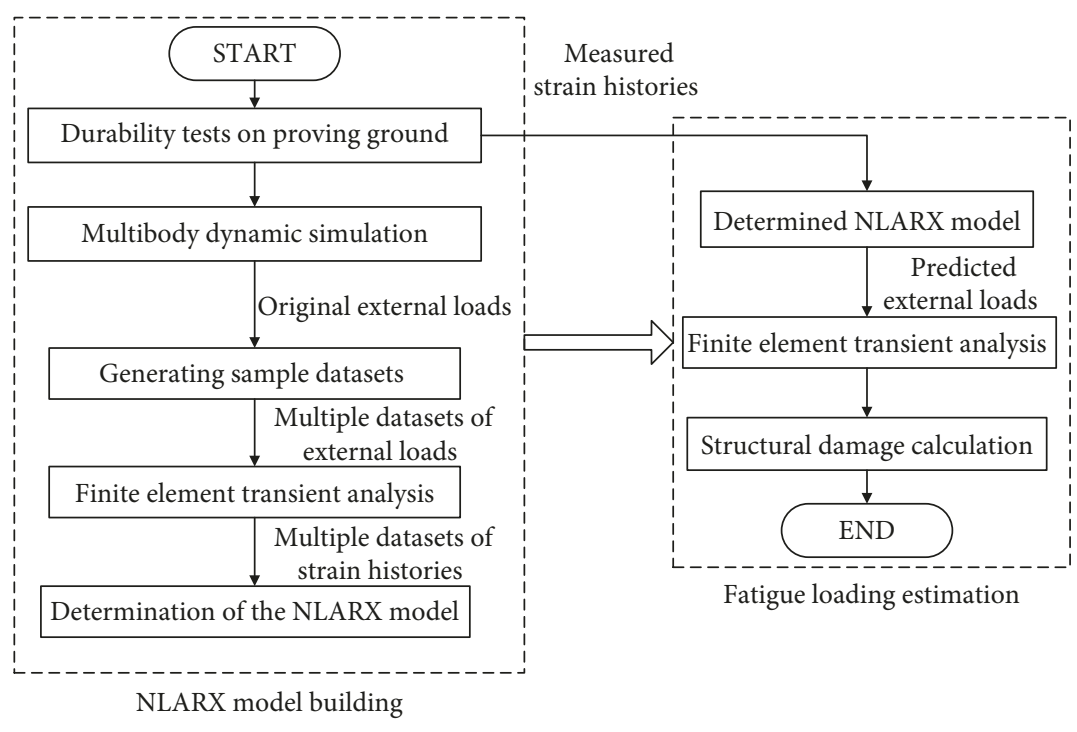

(b)

Figure 1: Framework of (a) semianalytical method; (b) the proposed approach.

loads of some components can be achieved by fixing the vehicle body and applying forces on the wheel spindles [14]. As a consequence, the most important task in the above studies is to improve the accuracy of multibody dynamic simulation. In their methodology, the accuracy of fatigue loading of automotive components depends on the external loads obtained by multibody dynamic simulation. However, with accurate external loads, significant error of fatigue loading cannot be eliminated, because of high correlation between them and inevitable error in finite element transient analysis $[5,15,16]$. Therefore, the aforementioned improved methods have limited ability to enhance the accuracy of fatigue loading.

To obtain accurate fatigue loading, Klemenc et al. [17] proposed a novel solution based on a combined multilayer perceptron neural network, which is used to determine the relationship between the operating conditions and the corresponding loading spectra. In their study, when ideal assumptions are true, they could predict loading spectra under limited number of discrete operating conditions. As automotive components usually experience complex random operating conditions and have nonlinear dynamic characteristics, this approach may be impossible to be applied in real cases. Nevertheless, this study promoted the application of neural network in structural durability design [18-21].

Here, indicated by current studies, a novel approach is proposed to estimate fatigue loading of automotive components based on the combination of a recurrent neural network NLARX and simplified semianalytical method. In this paper, continuous strain histories are used as study subjects for two reasons. Firstly, strain-life fatigue damage method is widely used in automotive industry even in highcycle application [22]. Secondly, fatigue loading of automotive components is commonly measured by strain rosettes. As shown in Figure 1(b), the framework of our proposed approach consists of two major parts: NLARX model building and fatigue loading estimation. In the procedure of building NLARX model which is analogous to that of conventional semianalytical method, firstly, multibody dynamic simulation is used to obtain original external loads. Then, multiple datasets of external loads are generated by the modification of the simulated external loads. Subsequently, strain histories corresponding to the generated external loads are obtained by finite element transient analysis. Finally, as the sample database, both the datasets of external loads and strain histories are used to determine the architecture of NLARX model. In the procedure of estimating fatigue loading, firstly, the determined NLARX model outputs the corresponding external loads from the input of measured strain histories. Then, the distribution of fatigue loading of automotive component is obtained by finite element transient analysis. Finally, structural damage of automotive component is calculated.

In our proposed approach, the external loads, which are used for the calculation of actual fatigue loading, is offered by the determined NLARX model rather than multibody dynamic simulation. Due to the generalization ability of NLARX, the multibody dynamic simulation is endowed to a certain degree of error space. The fault-tolerant feature enables numerous simplifications of multibody model. As a result, our proposed approach is able to obtain accurate fatigue loading with much less effort in establishing multibody model. To demonstrate the superiority of our proposed approach, durability tests of a torsion beam rear suspension are conducted.

The remainder of this paper is organized as follows. In Section 2, theoretical background of the proposed approach is presented. Then, the proposed approach is applied to estimate the fatigue strain histories of a torsion beam rear suspension in Section 3. Finally, the proposed approach is assessed in Section 4. 


\section{Theoretical Background of the Proposed Approach}

2.1. NLARX Model. Artificial neural networks are used to map complex nonlinear relationship which is difficult to be described with specific physical model. Among various types of artificial neural networks, NLARX model has proved to be suitable for problem of nonlinearity and high dynamics [23-26]. As a recurrent neural network, NLARX model extracts nonlinear dynamics by feeding previous network outputs and inputs back into the input layer. To represent the nonlinear dynamics best, it enables the user to choose the required number of previous outputs and input time steps [23]. Figure 2 shows the structure of a typical NLARX model. Between the input layer and output layer, there exist time delay line (TDL) and multilayer perceptrons for nonlinear analysis.

The NLARX model is suitable for multi-input multioutput nonlinear system, which is common in the process of automotive durability design. However, there are few studies on it. In this paper, the input of an NLARX model is represented by $\mu(t)$, which is a set of normalized strain histories at measurement positions. The output $y(t)$ represents the normalized external loads. The formulation of the NLARX model is described as follows:

$$
\begin{aligned}
y(t)= & F\left(y_{1}(t-1), \ldots, y_{1}\left(t-a_{1}\right), y_{2}(t-1), \ldots,\right. \\
& y_{2}\left(t-a_{2}\right), \ldots, y_{O}(t-1), \ldots, y_{O}\left(t-a_{O}\right), \ldots, \\
& \mu_{1}(t), \ldots, \mu_{1}\left(t-b_{1}+1\right), \mu_{2}(t), \ldots, \\
& \left.\mu_{2}\left(t-b_{2}+1\right), \ldots, u_{I}(t), \ldots, u_{I}\left(t-b_{I}+1\right)\right),
\end{aligned}
$$

where $O$ and $I$ are the number of output node and input nodes, respectively; $a_{1}, a_{2}, \ldots, a_{O}$ are the numbers of output delay; $b_{1}, b_{2}, \ldots, b_{I}$ are the numbers of input delay; and $F$ is the transfer function between the input and output layers, determined by the structure of the multilayer perceptrons. There are one or more hidden layers of neurons in the multilayer perceptron structure. The output of each neuron in the hidden can be described as the following equation [27]:

$$
z_{j}=\sigma_{j}\left(\sum_{i=1}^{N_{p}} \omega_{i j} z_{i}+\theta_{j}\right),
$$

where $z_{j}$ is the output of the neuron in an instantaneous layer; $z_{i}$ the output of the preceding layer; $\omega_{i j}$ the weight parameter between the adjacent layers; $\theta_{j}$ the bias parameter; $\sigma_{j}$ the activation function; and $N_{\mathrm{p}}$ the total number of the neurons in the preceding layer. The relationship between the external loads and the corresponding strain histories is achieved by iteratively training the NLARX model, which is a process to minimize the cost function:

$$
\operatorname{MSE}=\frac{1}{2 M} \frac{1}{O} \sum_{j=1}^{O} \sum_{t=1}^{M}\left(y_{j}(t)-v_{j}(t)\right)^{2},
$$

where $y_{j}(t)$ represents the predicted normalized external loads from the NLARX model; $v_{j}(t)$ the normalized experimental values from road tests; and $M$ the total number of the training samples.

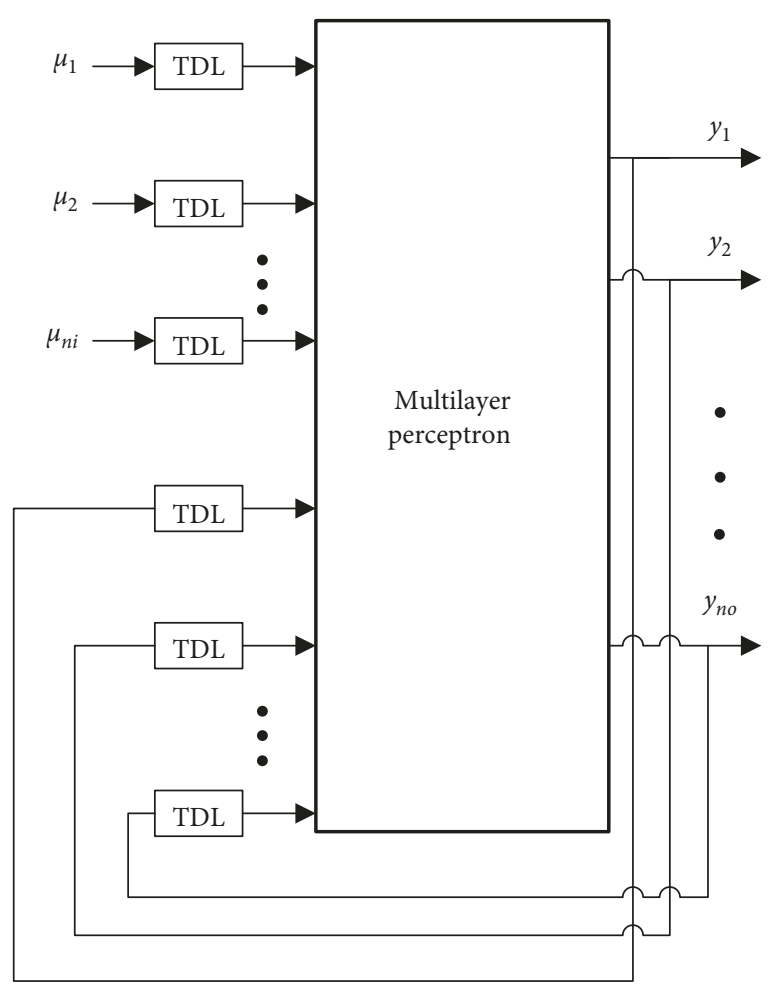

FIgURE 2: Canonical structure of an NLARX model.

The training of the NLARX model involves updating the weight parameters recurrently until the relationship between the inputs and the outputs is satisfactorily assessed. The details of the training process can be found in [27]. To build the accurate relationship between the external loads and their corresponding strain histories, various hyperparameters in the NLARX model have to be determined by multiple attempts, such as the number of delays for network inputs and outputs, the number of the hidden layers, and the number of hidden neurons.

In order to improve generalization ability and avoid overfitting during training of the NLARX model, the sample database is split into training datasets and testing datasets. The training datasets are selected based on the guideline of comprehensiveness and distinctiveness. The testing datasets, which are used to calibrate the NLARX model, are not a subset of the training sets. After training, the NLARX model reads the testing datasets and calculates an average error for them. In the end, the NLARX network is saved on the minimum error for testing datasets.

2.2. Simplification of the Flexible Multibody Models. In our proposed approach, the critical external loads corresponding to the experimental strain histories are obtained from an NLARX model rather than multibody dynamic simulation. As for multibody dynamic simulation, it is used to obtain a data sample for the NLARX model and determine the key external loads primarily responsible for structural damage. Due to its small effect on the estimation of structural strain histories, multibody dynamic simulation can be implemented based on extraordinarily simplified multibody model. 
For automotive bearing components, strain responses are mainly affected by vertical forces $[5,28]$. Therefore, it is assumed that simplified multibody models with only accurate characteristics in the vertical direction can make the simulated external loads close to real results.

\subsection{Measures for Assessing the Estimated Strain Histories.} Fatigue damage of automotive components is usually evaluated by using a strain-life fatigue damage method. Among several plastic strain models for strain-life assessment, Smith-Watson-Topper (SWT) model is widely used, whose expression is defined as follows [29, 30]:

$$
\sigma_{\max } \varepsilon_{\mathrm{a}} E=\left(\sigma_{\mathrm{f}}^{\prime}\right)^{2}\left(2 N_{\mathrm{f}}\right)^{2 b}+\sigma_{\mathrm{f}}^{\prime} \varepsilon_{\mathrm{f}}^{\prime} E\left(2 N_{\mathrm{f}}\right)^{b+c},
$$

where $\sigma_{\max }=\sigma_{\mathrm{m}}+\sigma_{\mathrm{a}}$ is the maximum stress, $\sigma_{\mathrm{m}}$ the mean stress, $\sigma_{\mathrm{a}}$ the stress amplitude, $\varepsilon_{\mathrm{a}}$ the total strain amplitude, $E$ the modulus of elasticity, $\sigma_{\mathrm{f}}^{\prime}$ the fatigue strength coefficient, $\varepsilon_{\mathrm{f}}^{\prime}$ the fatigue ductility coefficient, $b$ the fatigue strength exponent, $c$ fatigue ductility exponent, and $2 N_{\mathrm{f}}$ the number of reversals until fatigue. In the SWT model, cycle distributions of $\sigma_{\max }$ and $\varepsilon_{\mathrm{a}}$ are the two key factors if material property has been known. To extract them from continuous strain-time histories, the cycle distributions of $\varepsilon_{\mathrm{a}}$ and mean strain $\varepsilon_{\text {mean }}$ are firstly calculated by rain-flow counting method. Then, the cycle distributions of $\sigma_{\max }$ is calculated based on cyclic stress-strain curve.

In this paper, three measures are used to assess the difference between the estimated strain histories and the experimental data. Among them, two measures indicate the agreement of the cycle distributions of $\varepsilon_{\mathrm{a}}$ and $\varepsilon_{\text {mean }}$, which use the following expression:

$$
M_{\mathrm{d}}=\frac{\left\{\sum_{i=1}^{M_{l}}\left|\left(N_{\text {irpre }}-N_{\text {irexp }}\right) / N_{\text {irexp }}\right|\right\}}{N_{1}} \times 100 \%,
$$

where $N_{1}$ is the number of bins in the histogram for the cycle distributions of $\varepsilon_{\mathrm{a}}$ and $\varepsilon_{\text {mean }}, N_{\text {irpre }}$ the number of cycles of the $i$-th mean value or the $i$-th amplitude value for estimated strain histories, and $N_{\text {irexp }}$ the number of cycles of the $i$-th mean value or the $i$-th amplitude value for the experimental data. The third measure indicates the difference between the logarithm value of fatigue life cycles of the estimated strain histories and the experimental data as given below:

$$
F_{\mathrm{d}}=\left|\frac{\log _{10}\left(N_{\text {fpre }}\right)-\log _{10}\left(N_{\text {fexp }}\right)}{\log _{10}\left(N_{\text {fexp }}\right)}\right| \times 100 \%,
$$

where $N_{\text {fpre }}$ is the fatigue life cycles calculated from the estimated strain histories and is $N_{\text {fexp }}$ the fatigue life cycles calculated from the experimental data.

The closer the three measures are to zero, the stronger the relationship is. The three measures are able to reflect both local consistency and global consistency of the estimated strain histories with the experimental data. It is because that rain-flow counting matrix and fatigue life cycles are sensitive to both local extreme value and entire distribution of the strain histories.

\section{Estimation of Strain Histories for a Torsion Beam Rear Suspension}

3.1. Durability Tests and Simplified Multibody Dynamic Analysis. To experimentally demonstrate the performance of our proposed approach, durability tests of a torsion beam rear suspension are conducted at proving ground. Torsion beam suspension is widely used in nondriven rear axles for its economy and space saving. As the key load-bearing component in a vehicle, it is subjected to complex variable loads under service loads. Our target rear suspension consists of seven main welded parts (Figure 3), the material properties of which are shown in Table 1.

Durability tests are performed under different road profiles (Belgian, washboard and highway). To acquire data related to the operation conditions of the rear suspension, two multiwheel transducers (MTS WFT 205/55R16) and two accelerometers (BCX 1203F) are mounted on the wheel spindles to measure wheel forces, moments, and vertical acceleration, respectively. The strain histories at seven measurement positions are measured by strain gauges (KYOWA, $120 \Omega$ ) with $45^{\circ}$ internal angle of rosette (Figure 4). The experimental data are recorded with the use of a MTS 64-channel data acquisition instrument (eDAQ with nominal measurement error $0.03 \%$ ) in the sampling rate of $409.6 \mathrm{~Hz}$. For ease of comparison between the road collected data and estimated strain histories, maximum principal strain are also stored in eDAQ. The equation to calculate maximum principal strain is given as follows:

$$
\varepsilon_{1}=\frac{\varepsilon_{0^{\circ}}+\varepsilon_{90^{\circ}}}{2}+\frac{1}{2} \sqrt{\left(\varepsilon_{0^{\circ}}-\varepsilon_{90^{\circ}}\right)^{2}+\left(2 \varepsilon_{45^{\circ}}-\varepsilon_{0^{\circ}}-\varepsilon_{90^{\circ}}\right)^{2}},
$$

where $\varepsilon_{0^{\circ}}, \varepsilon_{45^{\circ}}, \varepsilon_{90^{\circ}}$ are the strain history of the three rosettes, respectively. To reduce measurement error, the rosettes are arranged in the form of Wheatstone bridge and the collected data are preprocessed by low-pass filter $(50 \mathrm{~Hz})$ operation, removing signal trend, and eliminating singular value.

In this study, multibody dynamic simulation is done based on simplified multibody model, in which springs with single degree of freedom are supposed to represent Macpherson front suspension and tires are omitted. Figure 5 shows the simplified multibody model and the complete fullsize multibody model for conventional semianalytical analysis. In both the two multibody models, the finite element model of torsion beam suspension is imported to ADAMS as flexible body part. Under the action of displacement signals on the wheel spindles, the responses (displacement and force histories) in the junction points between flexible and rigid body are output and then work as exciting signals in the following finite element transient analysis. Figure 6 shows the vertical wheel force from durability tests and multibody dynamic simulation. Both the simulated results from simplified multibody model and complete full-size model are distributed close to the experimental data. Table 2 and Figure 7 present the statistics characteristics and probability density distribution for the three sets of data, respectively. In Table 2, the values in the brackets are the relative increase compared with the experimental data. 


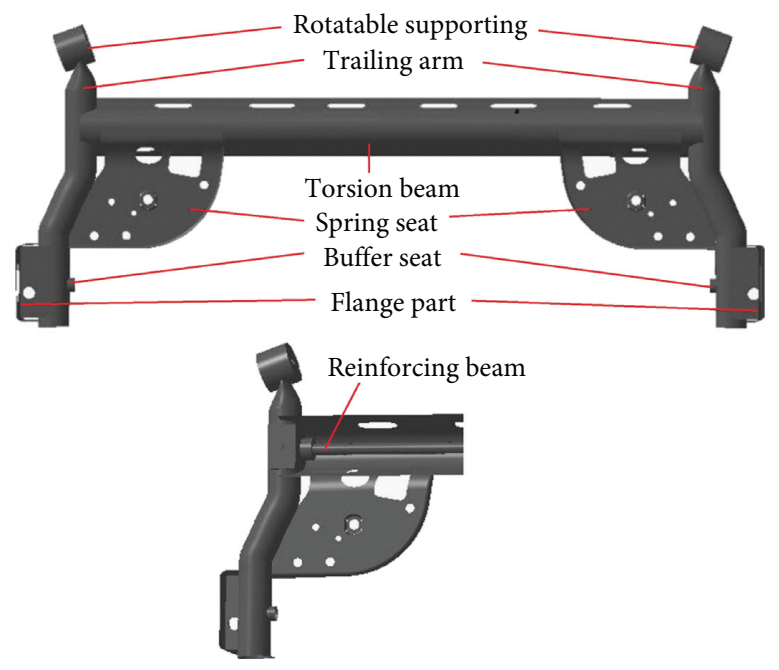

FIgURE 3: Target torsion beam rear suspension.

TABLE 1: Material properties for each part of the torsion beam suspension.

\begin{tabular}{|c|c|c|c|c|c|c|c|}
\hline & $\begin{array}{l}\text { Rotatable } \\
\text { supporting }\end{array}$ & $\begin{array}{c}\text { Trailing } \\
\text { arm }\end{array}$ & $\begin{array}{c}\text { Torsion } \\
\text { beam }\end{array}$ & $\begin{array}{c}\text { Spring } \\
\text { seat }\end{array}$ & $\begin{array}{c}\text { Buffer } \\
\text { seat }\end{array}$ & $\begin{array}{c}\text { Flange } \\
\text { part }\end{array}$ & $\begin{array}{c}\text { Reinforcing } \\
\text { beam }\end{array}$ \\
\hline Thickness (mm) & Solid & 4 & 4.5 & 4 & 10 & 6 & Solid \\
\hline Ultimate tensile strength ( $\mathrm{MPa})$ & 440 & 550 & 568 & 550 & 470 & 510 & 537 \\
\hline Yield strength $(\mathrm{MPa})$ & 235 & 345 & 432 & 430 & 310 & 416 & 305 \\
\hline Elasticity modulus (MPa) & $2.07 E 5$ & $2 E 5$ & $2 E 5$ & $2 E 5$ & $2 E 5$ & $2 E 5$ & $2.07 E 5$ \\
\hline Fatigue strength coefficient (MPa) & 2144 & 917 & 950 & 917 & 570 & 421 & 1517 \\
\hline Fatigue strength exponent & -0.179 & -0.095 & -0.095 & -0.095 & -0.09 & -0.09 & -0.14 \\
\hline Fatigue ductility coefficient & 2.145 & 0.26 & 0.117 & 0.662 & 0.03 & 0.13 & 0.312 \\
\hline Fatigue ductility exponent & -0.715 & -0.47 & -0.597 & -0.628 & -0.29 & -0.35 & -0.584 \\
\hline
\end{tabular}

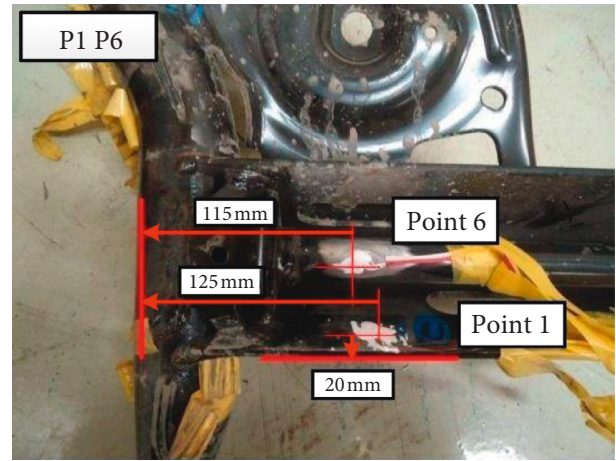

(a)

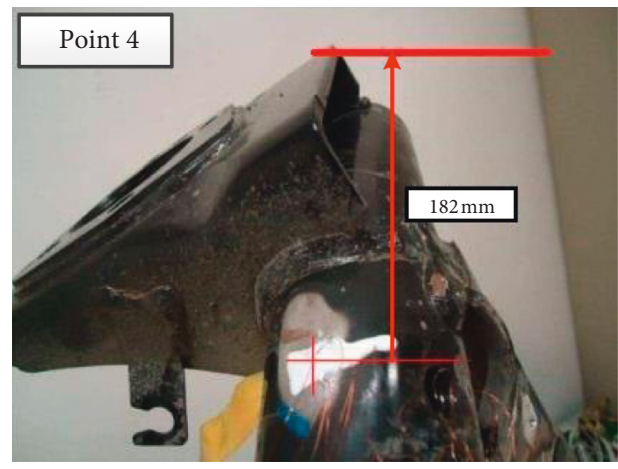

(c)

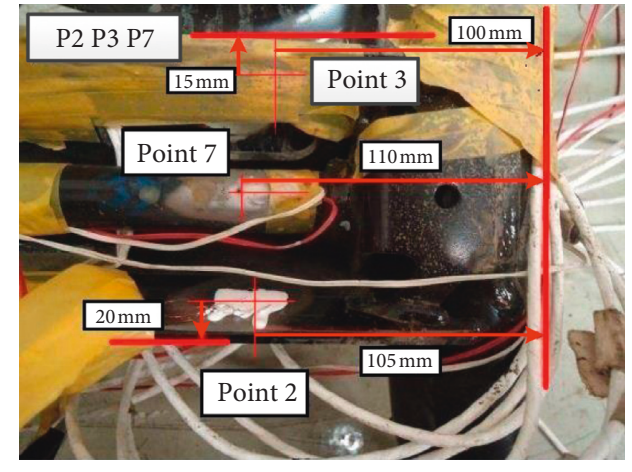

(b)

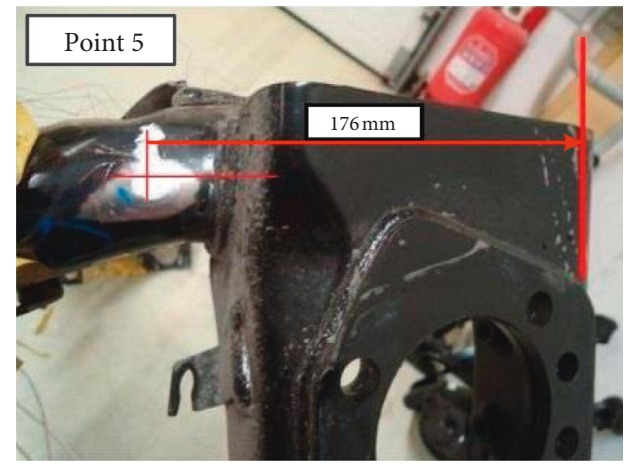

(d)

Figure 4: Measurement positions on the suspension. 


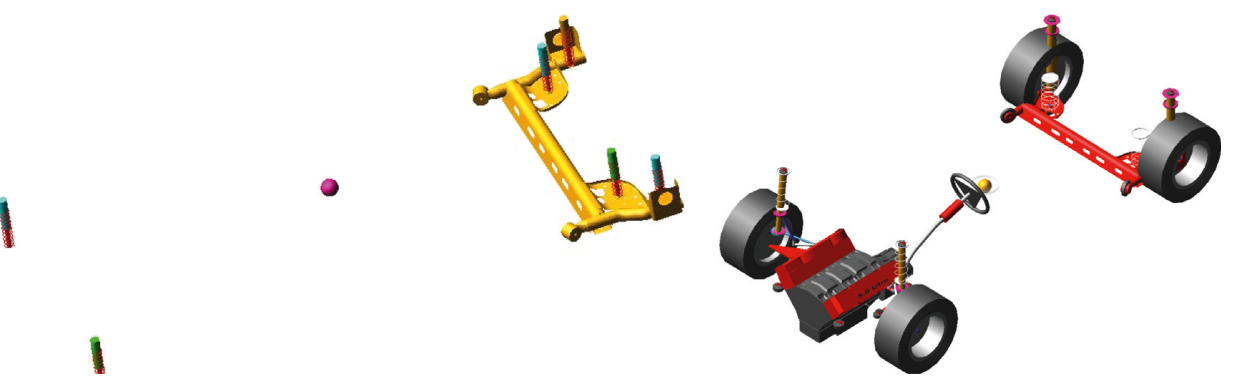

(a)

(b)

FIGURE 5: (a) Simplified multibody model; (b) complete full-size model.

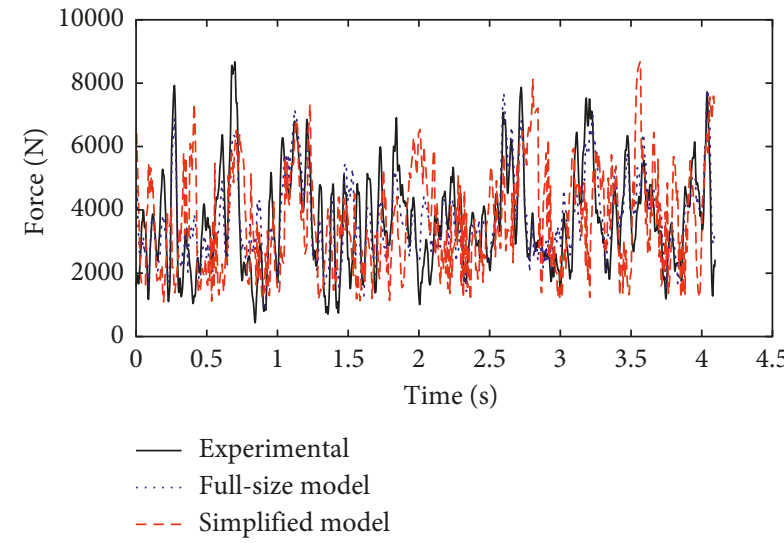

(a)

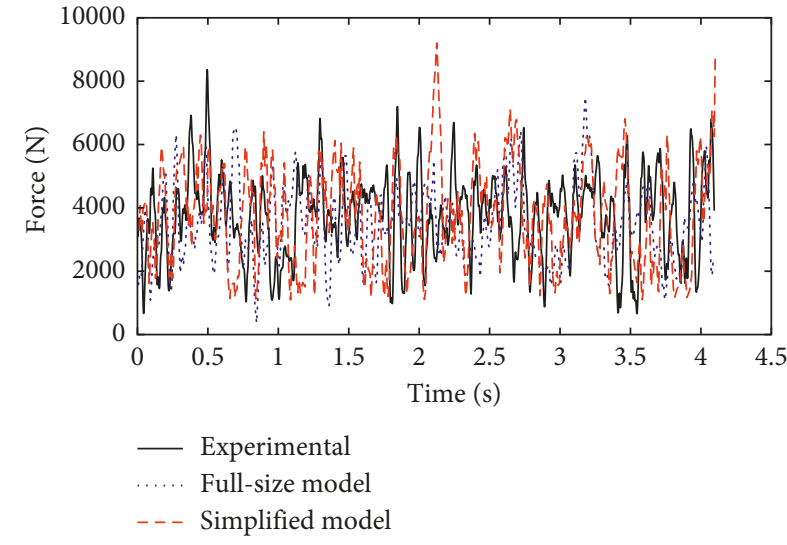

(b)

Figure 6: Vertical force of (a) left wheel; (b) right wheel.

TABLE 2: Statistics characteristics for vertical wheel force histories.

\begin{tabular}{lcccccc}
\hline & \multicolumn{2}{c}{ Mean value } & \multicolumn{2}{c}{ Standard deviation value } & \multicolumn{2}{c}{ Maximum value } \\
& Left & Right & Left & Right & Left & Right \\
\hline Experimental data & 3670 & 3767 & 1579 & 1301 & 8673 & 8361 \\
Full-size model & $3688(-0.49 \%)$ & $3482(-7.6 \%)$ & $1314(-16.8 \%)$ & $1236(-5 \%)$ & $7784(-10.3 \%)$ & $7510(-10.2 \%)$ \\
Simplified model & $3668(-0.054 \%)$ & $3807(1.1 \%)$ & $1731(9.6 \%)$ & $1608(23.6 \%)$ & $8774(1.16 \%)$ & $9290(11.1 \%)$ \\
\hline
\end{tabular}

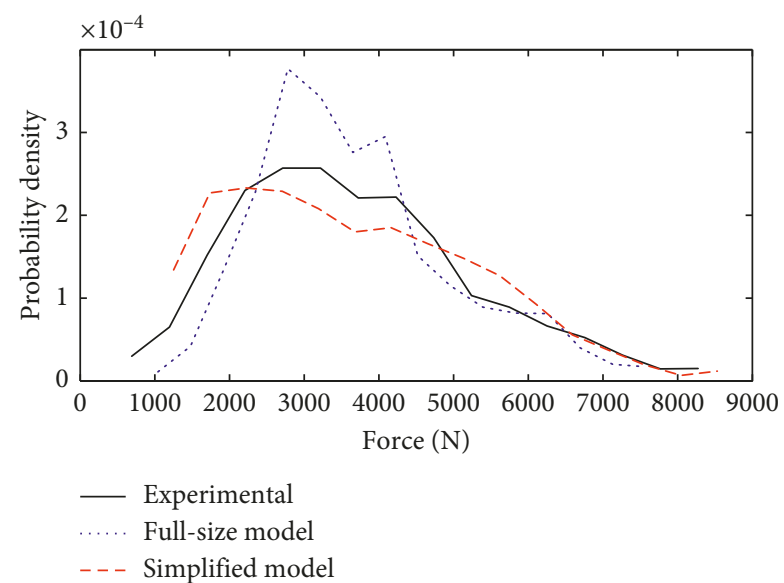

(a)

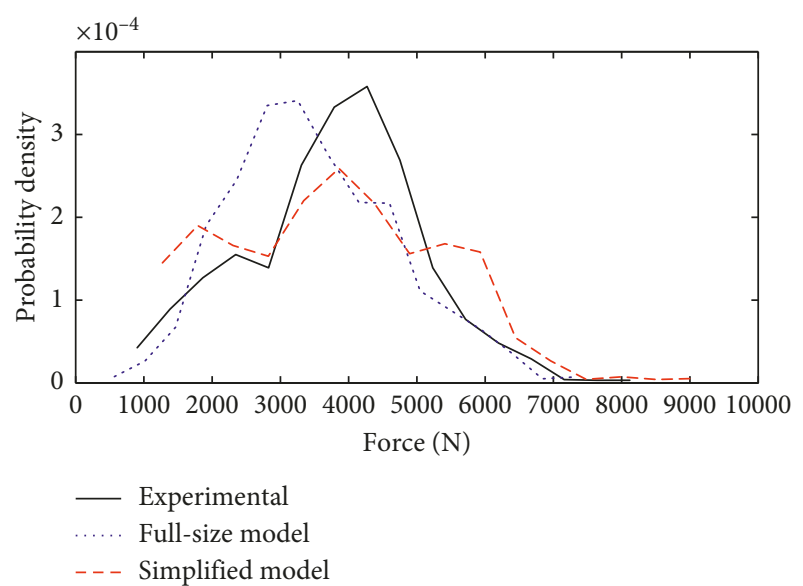

(b)

Figure 7: Probability density distribution: vertical force of (a) left wheel; (b) right wheel. 
As shown in Figure 6, the vertical wheel force from the simplified model is not as good as those from the complete full-size model in the aspect of wave shape because of the simplification of front suspension. However, their statistics characteristics are still close to those of the experimental data (Table 2). From Figure 7, it can be observed that the complete full-size model does not have obvious advantages over the simplified model. In this study, minimum data representing the operating conditions are extracted in consideration of analytical efficiency [23].

\subsection{Building the NLARX Model}

3.2.1. Selection of Sample Databases. The input of the NLARX model is set to the strain histories in the seven measurement positions. The external loads significantly influencing the strain responses of the rear suspension are selected as the output of the NLARX model. According to the research in [28], the vertical displacement at the spring seats is primarily responsible for strain response at the measurement positions.

The sample data of the vertical displacement are obtained based on the modification of the simulated external loads from simplified multibody dynamic simulation. More specifically, they are the linear combinations of the simulated external loads at spring seats and the experimental strain histories as follows:

$$
\begin{aligned}
& Y_{\text {lsample }}(t)=Y_{1}(t)+f S_{h}(t), \\
& Y_{\text {rsample }}(t)=Y_{\mathrm{r}}(t)+f S_{h}(t), \\
& 0.01 \leq f \leq 0.05, h=1,2, \ldots, 7,
\end{aligned}
$$

where $Y_{1}(t)$ and $Y_{\mathrm{r}}(t)$ are the vertical displacement at left spring seat and right spring seat obtained from multibody dynamic simulation, respectively, $S_{h}(t)$ is the experimental strain histories at the $h$-th measurement point, and $f$ the scale factor confined in the range of 0.01 to 0.05 . The small scale factor is chosen because even small changes of the vertical displacement at the spring seats can cause obvious differences of strain histories at the measurement positions. Because of their slight differences, the sample data of vertical displacement are not suitable as the outputs of an NLARX model. To solve this problem, $Y_{\text {lsample }}(t)$ and $Y_{\text {rsample }}(t)$ are preprocessed by subtracting denoised signals. In this study, the denoised signals are obtained by filtering $Y_{1}(t)$ and $Y_{\mathrm{r}}(t)$ with db5 3 level discrete wavelet transformation.

The sample data of strain responses corresponding to $Y_{\text {lsample }}(t)$ and $Y_{\text {rsample }}(t)$ are provided by finite element transient analysis. As structural failure did not occur until tens of thousands of kilometers in the proving ground tests and the experimental strain histories at the measurement positions are in the linear elastic range, the rear suspension is supposed to do not suffer from plastic deformation. Therefore, linear transient analysis is adopted to obtain simulated strain histories. The process of building the finite element model and making transient analysis are similar to the descriptions in researches [31-33]. In this study, the finite element model is composed primarily of quadrilateral shell element, including
59830 elements and 63249 nodes. The transient analysis is completed under the following boundary conditions: sixcomponent forces on the wheel spindles, three-directional displacement on the rotatable supportings, and vertical displacement on the spring seats and buffer seats. All the preprocessed vertical displacements and their corresponding strain histories are normalized as follows:

$$
\begin{aligned}
& X_{N}=\frac{X(t)-\overline{X(t)}}{\sigma_{X(t)}}, \\
& Y_{N}=\frac{Y(t)-\overline{Y(t)}}{\sigma_{Y(t)}},
\end{aligned}
$$

where $X_{N}$ and $Y_{N}$ are the normalized value of input strain histories and output external loads $Y(t)$, respectively; $\overline{X(t)}$ and $\overline{Y(t)}$ are the mean value for input strain histories and output vertical displacement, respectively; and $\sigma_{X(t)}$ and $\sigma_{Y(t)}$ are the standard deviation for input strain histories and output vertical displacement, respectively.

Considering the fact that the training of an NLARX model is slow, the number of training datasets is limited to a certain number. Moreover, to improve the prediction accuracy, the training datasets must cover the range of the experimental data as much as possible. In this study, 48 groups of the sample data are selected as training datasets for their extensive range, and the remaining 3 groups with significant differences as testing datasets.

\subsubsection{Determining the Architecture of the NLARX Model.} The selection of the hyperparameters is critical to the performance of NLARX model. Various NLARX models are built, varying along several main hyperparameters: the number of nonlinear hidden layers $\left(n_{h}\right)$, the number of neurons in each hidden layers $\left(n_{c}\right)$, the number of the input and output delay $\left(b_{i}\right.$ and $\left.a_{i}\right)$, and the type of the activation function in each hidden layers. For each NLARX model, they first accomplish calibration training on the training datasets. Then, they read the testing datasets and compute the corresponding output. There are hardly any specific reference theories or empirical formula for the selection of the aforementioned hyperparameters [27]. By trial and error, our optimal NLARX model with the lowest prediction error MSE for testing datasets is shown in Figure 8, whose hyperparameters settings are $n_{h}=2, n_{c}^{1}=n_{c}^{2}=30, b_{i}=10$, $a_{i}=4$. During the training of the optimal NLARX model, the weight parameters and bias parameters are updated by stochastic gradient decent with momentum and five-fold cross validation method. The learning rate $\eta$ and momentum $\gamma$ are set to $1 \mathrm{E}-10$ and 0.9 .

3.2.3. Result of the NLARX Model. The optimal NLARX model is applied to predict the relationship between critical external loads and their corresponding strain histories. In the process of training (less than 50 minutes), this NLARX model can achieve the goal $(\mathrm{MSE}=0.16)$ within 50 iterations. Figures 9 and 10 show the predicted results of the NLARX model for training datasets and testing datasets. 


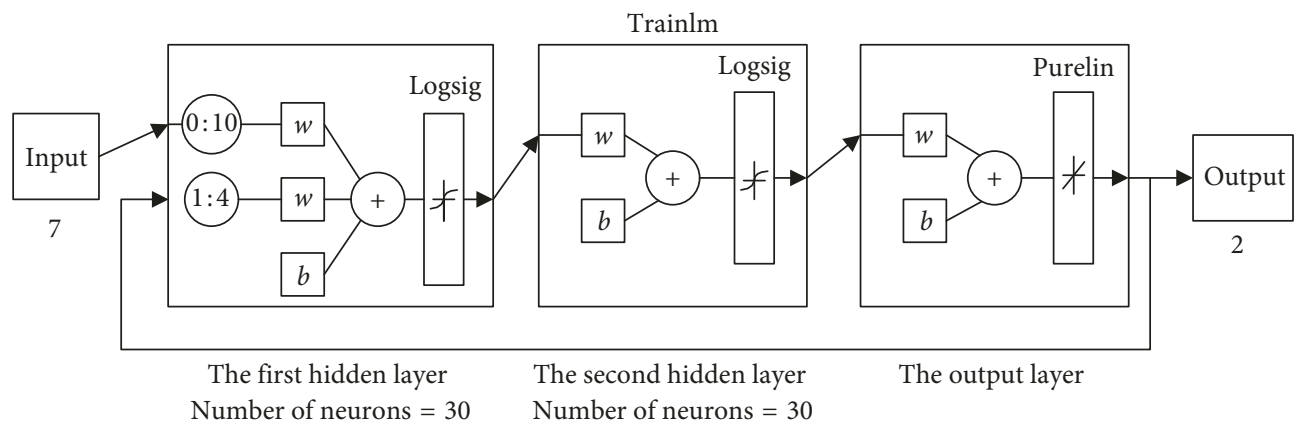

Figure 8: Architecture of the optimal NLARX model.
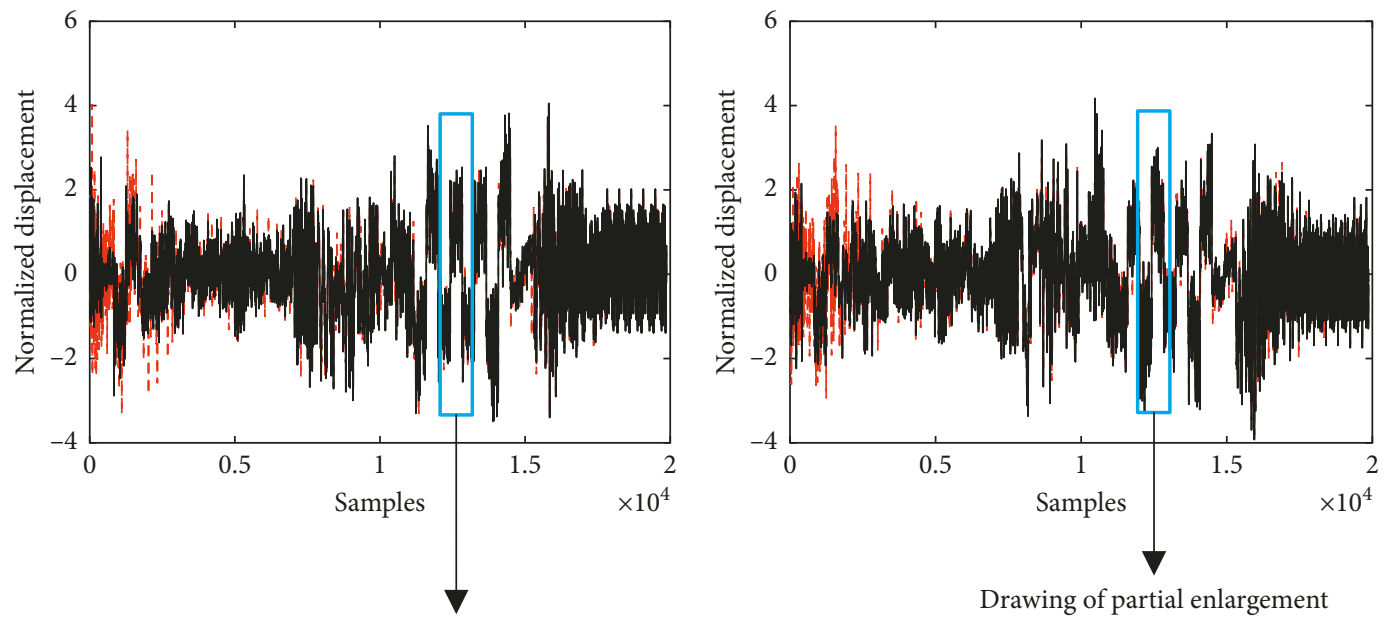

Drawing of partial enlargement

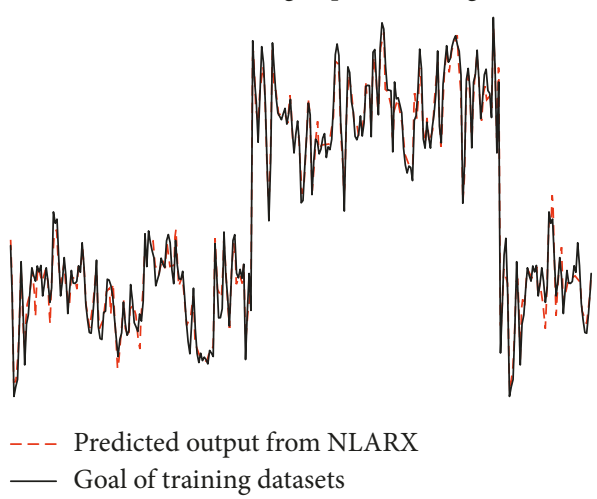

(a)

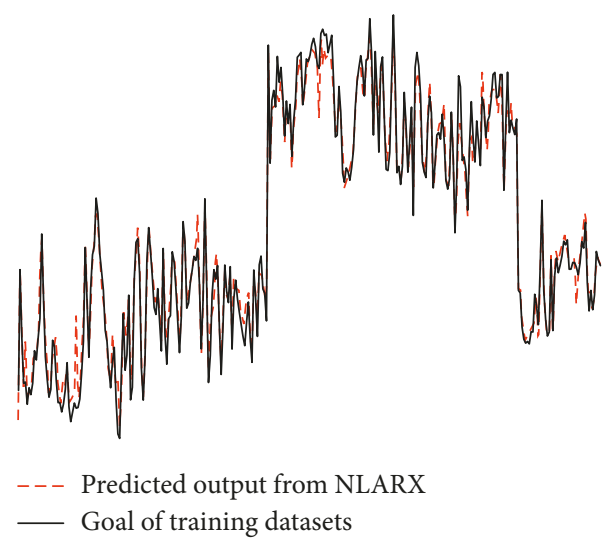

(b)

FIGURE 9: NLARX results for training datasets: (a) left external loads; (b) right external loads.

The recurrent coefficient $R$-square $\left(R^{2}\right)$ is used to evaluate the performance of the predicted external loads from the optimal NLARX model. The closer $R^{2}$ is to one, the stronger the relationship is. The predicted results are as follows: for the training dataset $R_{\mathrm{LEFTtrain}}^{2}=0.926, R_{\mathrm{RIGHT} \text { train }}^{2}=0.90$; for the testing datasets, $R_{\text {LEFTtest }}^{2}=0.822, R_{\text {RIGHTtest }}^{2}=0.801$.

For the training datasets, the NLARX model predicts the external loads very well other than the beginning of the time series values. The relative greater error of the beginning parts is inevitable for recurrent neural network, caused by the incomplete closed-loop recurrent back propagation training.
For the testing datasets, the recurrent coefficient $\left(R^{2}\right)$ between the predicted and original external loads is slightly less than that for the training data-sets. However, considering the large range of the testing datasets and the obvious differences between the training datasets and testing datasets, the NLARX model proves to be with high generalization ability.

For the training results and testing results from the NLARX model, the results on the left side are slightly more accurate than the results on the right side. It is because the left external loads for training of the NLARX network are 


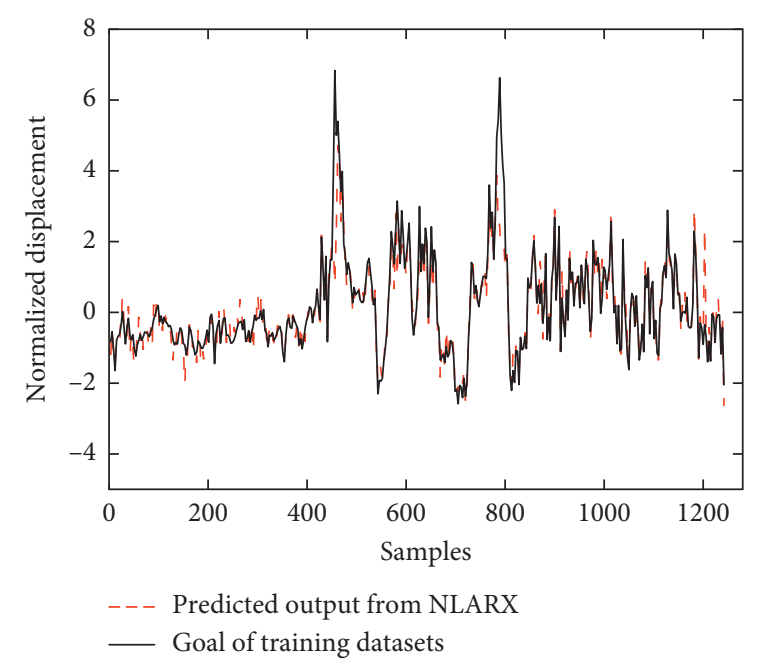

(a)

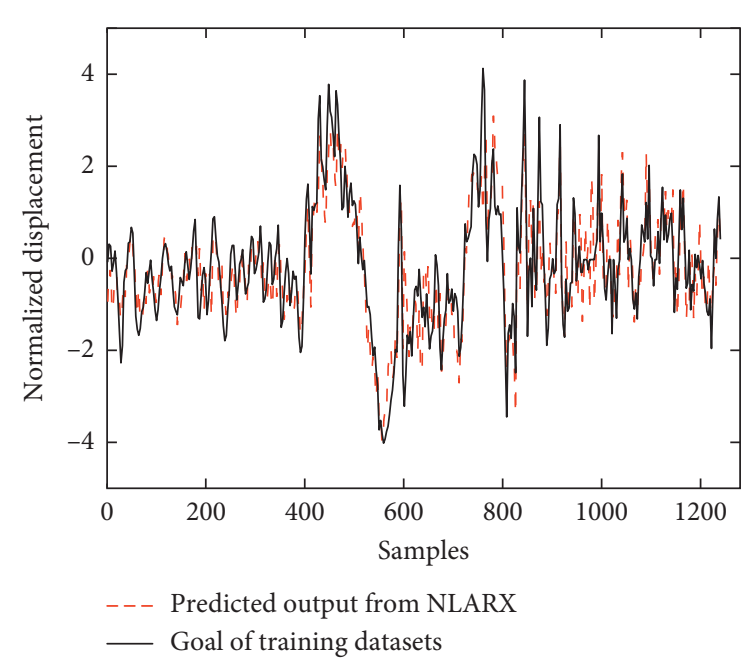

(b)

Figure 10: NLARX results for testing datasets: (a) left external loads; (b) right external loads.

more irregular. Specifically, the amplitude and variance of the left external loads are $2.91 \%$ and $6.42 \%$ smaller than those of the right external loads. The subtle differences are mainly caused by the differences of road profiles on each wheel.

After the optimal NLARX model is trained and tested by the sample database, the predicted external loads corresponding to experimental strain histories are calculated as shown in Figure 11. The simulation results at the spring seats, obtained from the full-size multibody model and simplified multibody model, are very close and can reflect the trend of the results from the NLARX. Furthermore, the local extrema of the external loads, which are difficult to be obtained from multibody models as the result of modeling error and measuring error, can be predicted by the NLARX model.

The predicted external loads from the NLARX model are applied to the finite element model of the torsion beam rear suspension for transient analysis. Figure 12 shows the transient responses at the measurement positions versus the experimental data and the strain histories by conventional semianalytical method. It is obvious that the estimated strain histories using the proposed approach are much closer to the experimental data when compared with those using conventional semianalytical method. Moreover, it can be found that the distribution of the local extrema of the external loads has great effects on the strain histories at the measurement positions.

In this study, the three measures $M_{\mathrm{d}}^{\text {mean }}, M_{\mathrm{d}}^{\text {amplitude }}$, and $F_{\mathrm{d}}$ are used to evaluate the quality of the estimated strain histories. For strain histories at the measurement positions, both their rain-flow matrices and fatigue life cycles are calculated by using nCodeGlyph Works software. The cycle distributions of $\varepsilon_{\mathrm{a}}$ and $\varepsilon_{\text {mean }}$, obtained from rain-flow matrices, are illustrated by histogram with eight bins (Figure 13). Compared with the conventional semianalytical method, the proposed approach is able to improve the estimation accuracy of cycle distributions of $\varepsilon_{\mathrm{a}}$ and $\varepsilon_{\text {mean }}$, especially when the values of $\varepsilon_{\mathrm{a}}$ and $\varepsilon_{\text {mean }}$ are large. Table 3 shows the agreement between the estimated strain histories and the experimental data assessed by the three measures. As expected, the proposed approach can achieve much higher estimation precision than conventional semianalytical method. These results further demonstrate that the welltrained NLARX network holds good generalization ability, because the experimental strain histories are not in the domain of the training and testing data-sets.

\section{Conclusion}

Fatigue loading contributes significantly to the fatigue life assessment of automotive components. To accurately estimate the fatigue loading, a novel approach is proposed based on the combination of an NLARX model and simplified semianalytical method. In the proposed approach, firstly, semianalytical method based on simplified multibody model is used to generate sample database for NLARX model training and testing. Then, under the excitation of the external load obtained from the well-trained NLARX model, strain histories in continuous time series can be achieved by finite element transient analysis. In the task of estimating strain histories for a torsion beam rear suspension, the proposed approach can achieve striking accuracy. Compared with conventional semianalytical method, the proposed approach offers much higher performance of the estimated strain histories according to three objective measures.

In our study case, the strong correlation between the estimated strain histories and the experimental data shows the rationality of simplifying multibody model and the reliability of an NLARX model. Consequently, the proposed approach is able to improve the fatigue life prediction, the cost control, and modeling efficiency in the process of durability design for automotive components. 


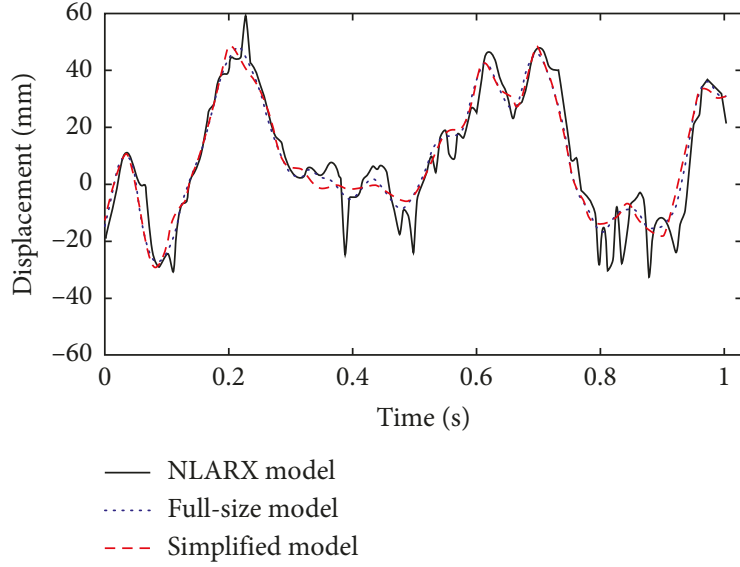

(a)

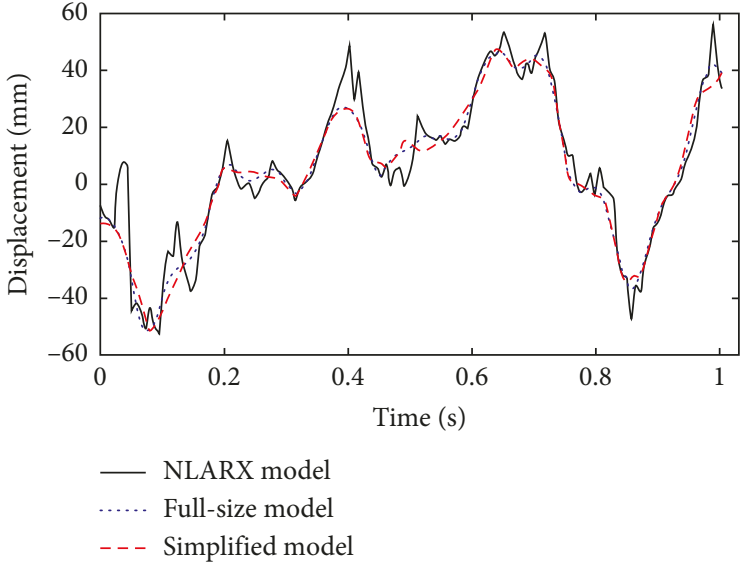

(b)

Figure 11: Vertical displacement histories: (a) at the left spring seats; (b) at the left spring seats.

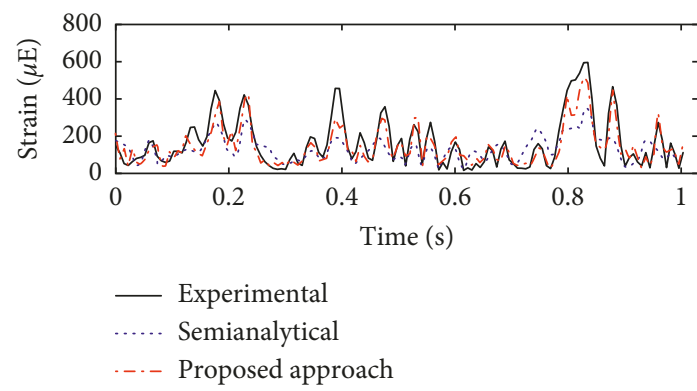

(a)

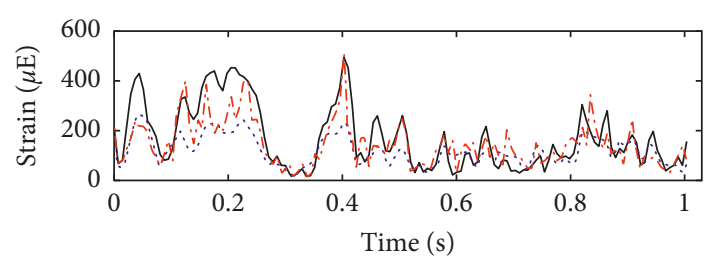

_... Experimental
.... Semianalytical
..- Proposed approach

(c)

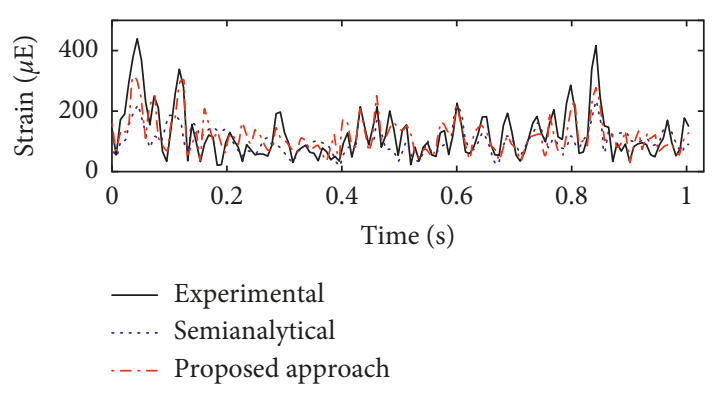

(e)

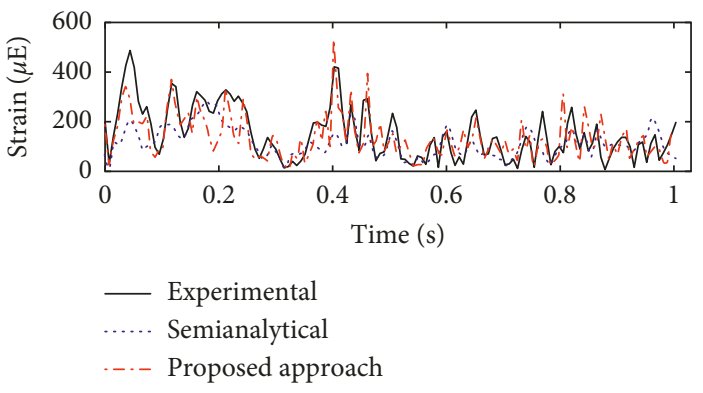

(b)

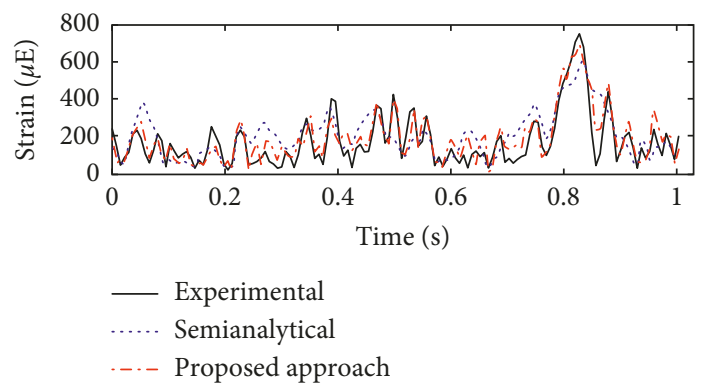

(d)

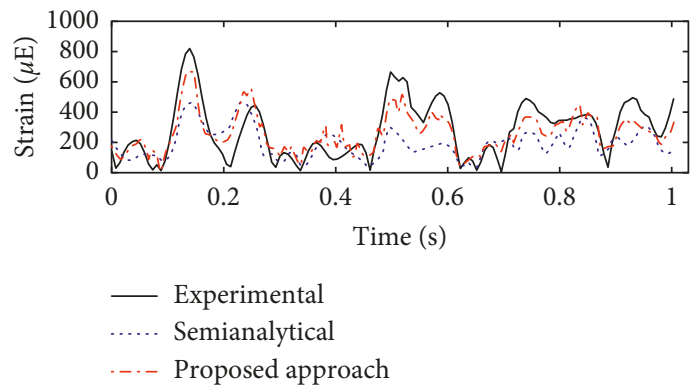

(f)

Figure 12: Continued. 


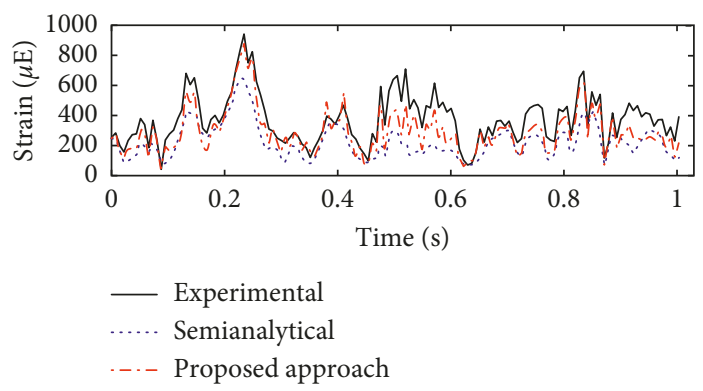

(g)

Figure 12: Strain histories at: (a) Point 1; (b) Point 2; (c) Point 3; (d) Point 4; (e) Point 5; (f) Point 6; (g) Point 7.

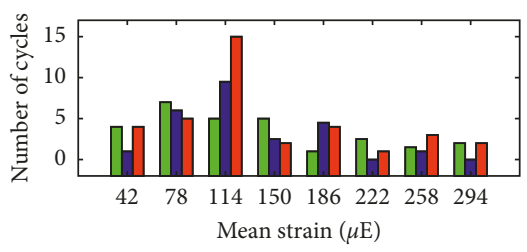

$\square$ Experimental

Semianalytical

Proposed approach

(a)

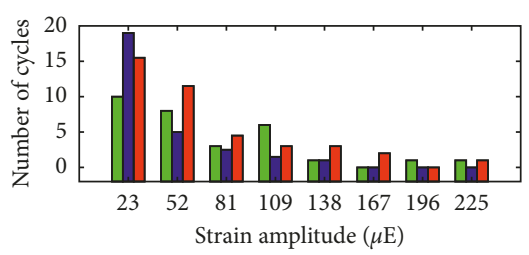

Experimental

Semianalytical

Proposed approach

(d)

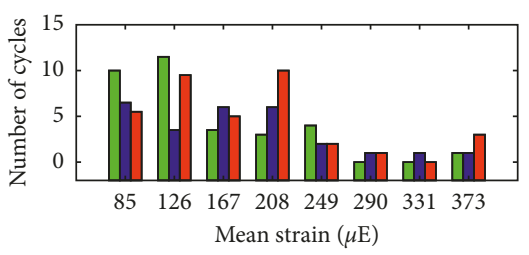

Experimental

Semianalytical

Proposed approach

(g)

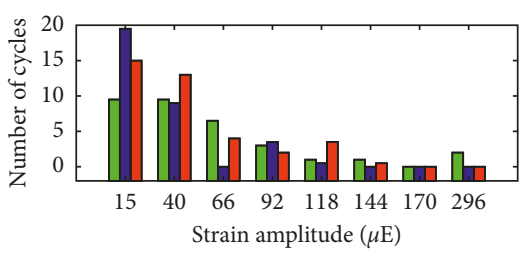

$\square$ Experimental

Semianalytical

$\square$ Proposed approach

(j)

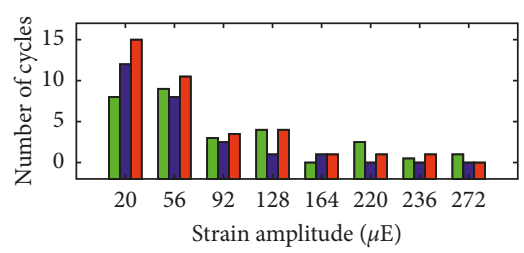

$\square$ Experimental

Semianalytical

$\square$ Proposed approach

(b)

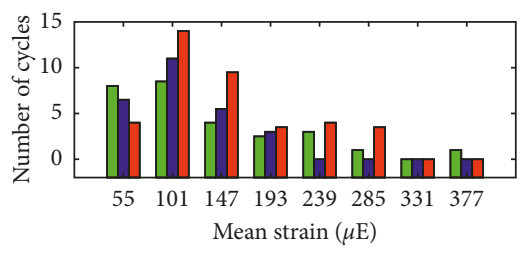

$\longleftarrow$ Experimental

Semianalytical
$\square$ Proposed approach

(e)

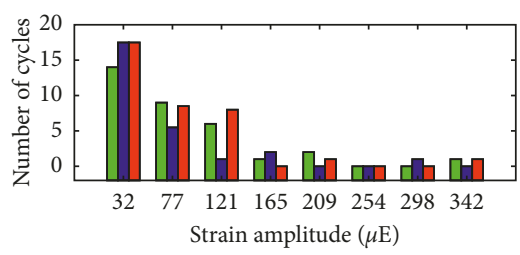

$\sqsubset$ Experimental

Semianalytical

$\square$ Proposed approach

(h)

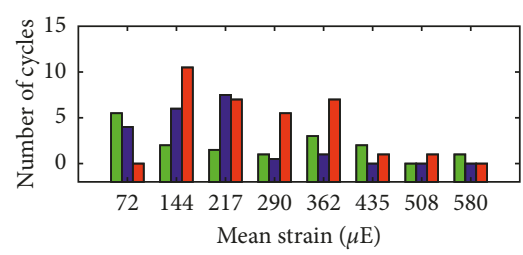

$\square$ Experimental
Semianalytical
$\square$ Proposed approach

(k)

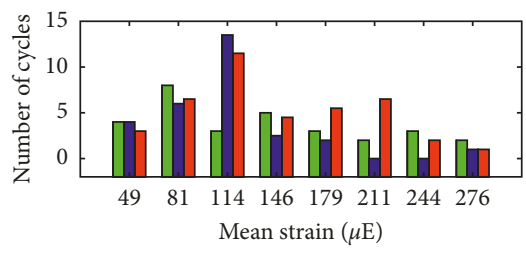

$\square$ Experimental

Semianalytical

ए Proposed approach

(c)

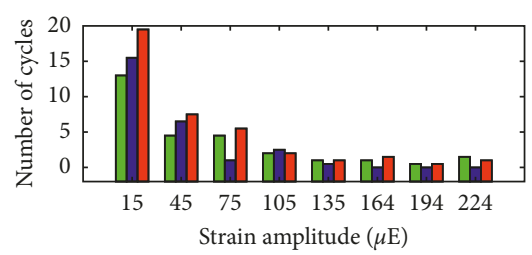

שxperimental

Semianalytical

$\square$ Proposed approach

(f)

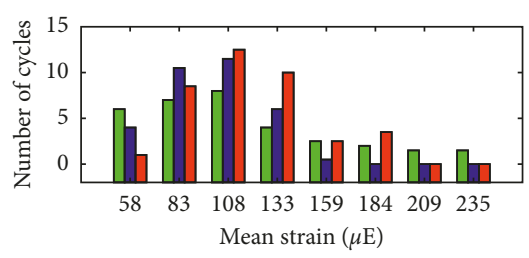

Experimental

Semianalytical

$\square$ Proposed approach

(i)

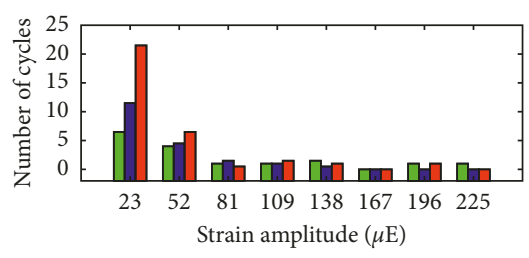

$\square$ Experimental

- Semianalytical

Proposed approach

(1)

FIgURE 13: Continued. 


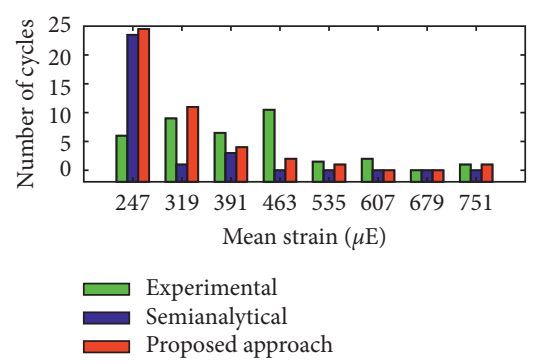

(m)

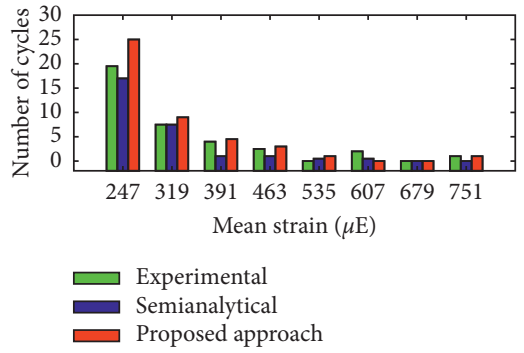

(n)

Figure 13: Cycle distributions of $\varepsilon_{\mathrm{a}}$ and $\varepsilon_{\text {mean }}$ : (a) and (b) at Point 1; (c) and (d) at Point 2; (e) and (f) at Point 3; (g) and (h) at Point 4; (i) and (j) at Point 5; (k) and (l) at Point 6; (m) and (n) at Point 7.

TABLE 3: Quality of the estimated strain histories.

\begin{tabular}{ccccccccc}
\hline Point no. & & Point 1 & Point 2 & Point 3 & Point 4 & Point 5 & Point 6 & Point 7 \\
Experimental life cycles & $3.22 E 10$ & $2.25 E 11$ & $1.76 E 11$ & $3.77 E 9$ & $6.22 E 11$ & $1.35 E 9$ & $4.91 E 8$ \\
\hline \multirow{3}{*}{ Proposed approach } & $M_{\mathrm{d}}^{\text {mean }}$ & 29.75 & 40 & 59 & 13.22 & 36.06 & 44 \\
& $M_{\mathrm{d}}^{\text {amplitude }}$ & 32.14 & 32.59 & 40.83 & 21.11 & 38.40 & 45.33 & 21.39 \\
& $F_{\mathrm{d}}$ & 7.02 & 2.88 & 0.02 & 3.51 & 13.54 & 10.15 & 2.80 \\
\hline \multirow{3}{*}{ Semianalytical } & $M_{\mathrm{d}}^{\text {mean }}$ & 64.96 & 60.33 & 69.34 & 51.28 & 64.33 & 51.33 & 70.75 \\
& $M_{\mathrm{d}}^{\text {amplitude }}$ & 58.29 & 67.87 & 64.17 & 46.67 & 58.45 & 54.57 & 58.33 \\
& $F_{\mathrm{d}}$ & 21.05 & 22.77 & 26.14 & 9.88 & 27.19 & 28.58 & 19.18 \\
\hline
\end{tabular}

In the future work, more training samples and more advanced neural network model will be explored to obtain more accurate fatigue loading.

\section{Data Availability}

The data used to support the findings of this study are available from the corresponding author upon request.

\section{Conflicts of Interest}

The authors declare that they have no conflicts of interest.

\section{Acknowledgments}

The experimental tests were supported by National Automobile Quality Supervision and Test Center (Xiangyang). This research is supported by National Key Technologies R\&D Program (grant no. 2015BAG08B02).

\section{References}

[1] E. Habtour, M. Paulus, A. Dasgupta et al., "Modeling approach for predicting the rate of frequency change of notched beam exposed to Gaussian random excitation," Shock and Vibration, vol. 2014, Article ID 164039, 11 pages, 2014.

[2] L. H. Zhao, H. C. Cai, T. Wang et al., "Durability assessment of automotive structural under random variable amplitude loading," Advances in Mechanical Engineering, vol. 10, no. 4, pp. 1-8, 2018.

[3] C. Braccesi, F. Cianetti, G. Lori, and D. Pioli, "Random multiaxial fatigue: a comparative analysis among selected frequency and time domain fatigue evaluation methods," International Journal of Fatigue, vol. 74, pp. 107-118, 2015.

[4] J. M. D. Pioli, I. L. D. E. Santo, and A. A. D. Oliveila, "A Semianalytical method to generate load cases for CAE durability using virtual vehicle prototypes," in Proceedings of the SAE Brazil 2003 Congress and Exhibit, pp. 3667-3672, Sao Paulo, Brazil, November 2003.

[5] S. H. Lin, C. G. Cheng, C. Y. Liao et al., "Experiment and CAE Analyses for suspension under durability road load conditions," in Proceedings of the SAE 2006 Word Congress and Exhibition, pp. 1624-1630, Detroit, MI, USA, April 2006.

[6] K. J. Jun, T. W. Park, S. H. Lee, S. P. Jung, and J. W. Yoon, "Prediction of fatigue life and estimation of its reliability on the parts of an air suspension system," International Journal of Automotive Technology, vol. 9, no. 6, pp. 741-747, 2008.

[7] C. Mi, Z. Gu, Q. Yang, and D. Nie, "Frame fatigue life assessment of a mining dump truck based on finite element method and multibody dynamic analysis," Engineering Failure Analysis, vol. 23, pp. 18-26, 2012.

[8] Z. Nie, C. Mi, Y. Wang, and J. Jiang, "A-type frame fatigue life estimation of a mining dump truck based on modal stress recovery method," Engineering Failure Analysis, vol. 26, pp. 89-99, 2012.

[9] M. Jiang, A. Dhir, R. Ranganathan et al., "A new tire model for road loads simulation: full vehicle validation," in Proceedings of the SAE 2004 Word Congress and Exhibition, pp. 15791583, Detroit, MI, USA, March 2004.

[10] H. Haga, "Evaluation method for road load simulation using a tire model and an applied example," in Proceedings of the SAE 2006 Word Congress and Exhibition, pp. 1256-1265, Detroit, MI, USA, April 2006.

[11] M. Baecker, A. Gallrein, and H. Haga, "A tire model for very large tire deformation and its application in very severe events," in Proceedings of the SAE 2010 Word Congress and Exhibition, pp. 142-151, Detroit, MI, USA, April 2010.

[12] P. Jayakumar, D. Wang, and S. Cline, "Issues of estimating powertrain mounts loads using measured accelerations and drive toraues for durability events," in Proceedings of the SAE 2005 Word Congress and Exhibition, pp. 626-130, Detroit, MI, USA, April 2005. 
[13] C. Cao and T. Medepalli, "Spindle-based engine mount load analysis-prediction and correlation," in Proceedings of the SAE 2007 Word Congress and Exhibition, pp. 1203-1207, Detroit, MI, USA, April 2007.

[14] L. G. Liu, X. F. Ran, and L. Li, "Hybrid vehicle road loads simulation and correlation," in Proceedings of the SAE 2007 Word Congress and Exhibition, pp. 1199-1202, Detroit, MI, USA, April 2007.

[15] S. Kangde, V. Shitole, and A. K. Sahu, "Suspension strain correlation using flex bodies in $\mathrm{MBD}$," in Proceedings of the SAE 2014 Word Congress and Exhibition, pp. 763-774, Detroit, MI, USA, April 2014.

[16] X. Wang, W. Xu, Y. Huang, M. Zhong, and H. Fan, "Simulation of the vertical bending fatigue test of a five-link rear axle housing," International Journal of Automotive Technology, vol. 13, no. 6, pp. 923-932, 2012.

[17] J. Klemenc and M. Fajdiga, "Predicting smoothed loading spectra using a combined multilayer perceptron neural network," International Journal of Fatigue, vol. 28, no. 7, pp. 777-791, 2006.

[18] P. Limbourg, "Approximating failure distributions from similar components using artificial neural networks," in Proceedings of the European Safety and Reliability Conference, pp. 911-919, Estoril, Portugal, September 2006.

[19] M. Kutylowska, "Neural network approach for failure rate prediction," Engineering Failure Analysis, vol. 47, pp. 41-48, 2015.

[20] S. Balakrishnan and R. C. Kharul, "Accurate estimation of time histories for improved durability prediction using artificial neural network," in Proceedings of the SAE 2012 Word Congress and Exhibition, pp. 23-33, Detroit, MI, USA, April 2012.

[21] H. Nbwangwa, P. S. Heyns, H. G. A. Breytenbach et al., "Reconstruction of road defects and road roughness classification using artificial neural network simulation and vehicle dynamic responses: application to experimental data," Journal of Terramechanics, vol. 53, pp. 1-18, 2014.

[22] R. Basan, M. Franulović, I. Prebil, and N. Črnjarić-Žic, "Analysis of strain-life fatigue parameters and behabiour of different groups of metallic materials," International Journal of Fatigue, vol. 33, no. 3, pp. 484-491, 2011.

[23] J. M. Črnjarić-Žic, B. Maass, and R. Stobart, "Minimum data requirement for neural networks based on power spectral density analysis," IEEE Transactions on Neural Networks, vol. 23, no. 4, pp. 587-595, 2012.

[24] J. M. Deng, B. Maass, and R. Stobart, "Single NLARX model for particulate matters prediction of diesel engines," in Proceedings of the 18th IFAC world congress, pp. 10641-10646, Milano, Italy, September 2011.

[25] J. Deng, S. Zhong, and A. Ordys, "Robustness and accuracy test of particular matter prediction based on neural networks," Communications and Network, vol. 5, no. 2, pp. 53-59, 2013.

[26] J. M. Deng, "Dynamic neural networks with hybrid structures for nonlinear system identification," Engineering Applications of Artificial Intelligence, vol. 26, no. 1, pp. 281-292, 2013.

[27] M. T. Hagan, H. B. Demuth, and M. H. Beale, Neural Network Design, Martin Hagan Press, Oklahoma, OK, USA, 2002.

[28] L. H. Zhao, S. L., Zheng, and J. Z. Feng, "Failure mode analysis of torsion beam rear suspension under service condition," Engineering Failure Analysis, vol. 36, pp. 1533-1541, 2013.

[29] J. Wannenburg, P. Stephan Heyns, and A. D. Raath, “Application of a fatigue equivalent static load methodology for the numerical durability assessment of heavy vehicle structures," International Journal of Fatigue, vol. 31, no. 10, pp. 1541-1549, 2009.

[30] W. Bedkowski, "Assessment of the fatigue life of machine components under service loading: a review of selected problems," Journal of Theoretical and Applied Mechanics, vol. 52, no. 2, pp. 443-458, 2014.

[31] X. F. Wang and X. G. Zhang, "Simulation of dynamic cornering fatigue test of a steel passenger car wheel," International Journal of Fatigue, vol. 32, no. 2, pp. 434-442, 2014.

[32] M. Firat, R. Kozan, M. Ozsoy, and O. H. Mete, "Numerical modeling and simulation of wheel radial fatigue tests," Engineering Failure Analysis, vol. 16, no. 5, pp. 1533-1541, 2009.

[33] N. A. Mete, S. Abdullah, and A. K. Ariffin, "Effective strain damage model associated with finite element modelling and experimental validation," International Journal of Fatigue, vol. 36, no. 1, pp. 194-205, 2012. 


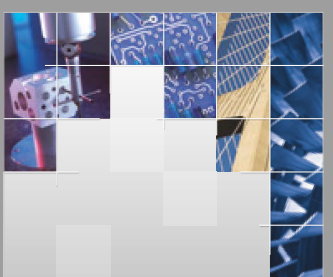

\section{Enfincering}
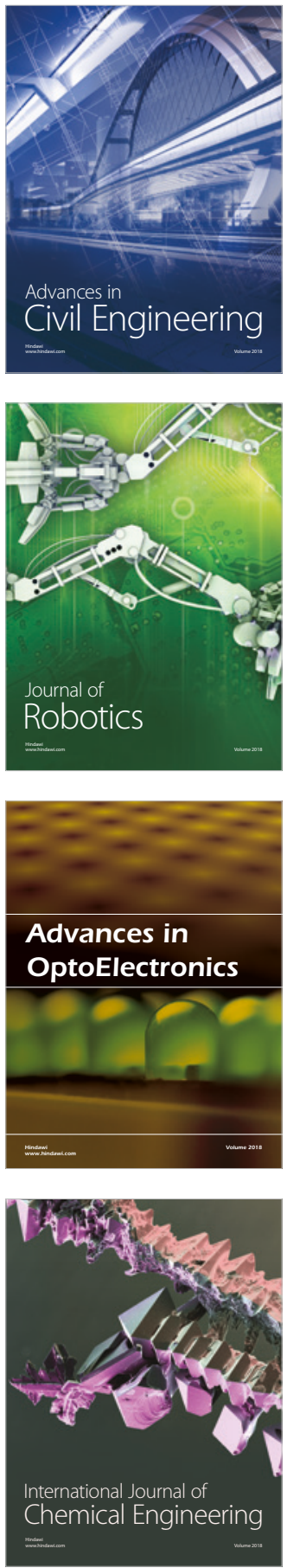

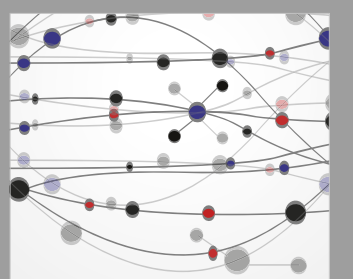

\section{Rotating \\ Machinery}

The Scientific World Journal

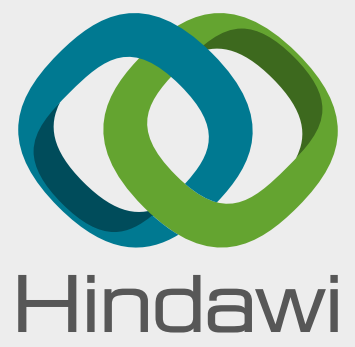

Submit your manuscripts at

www.hindawi.com
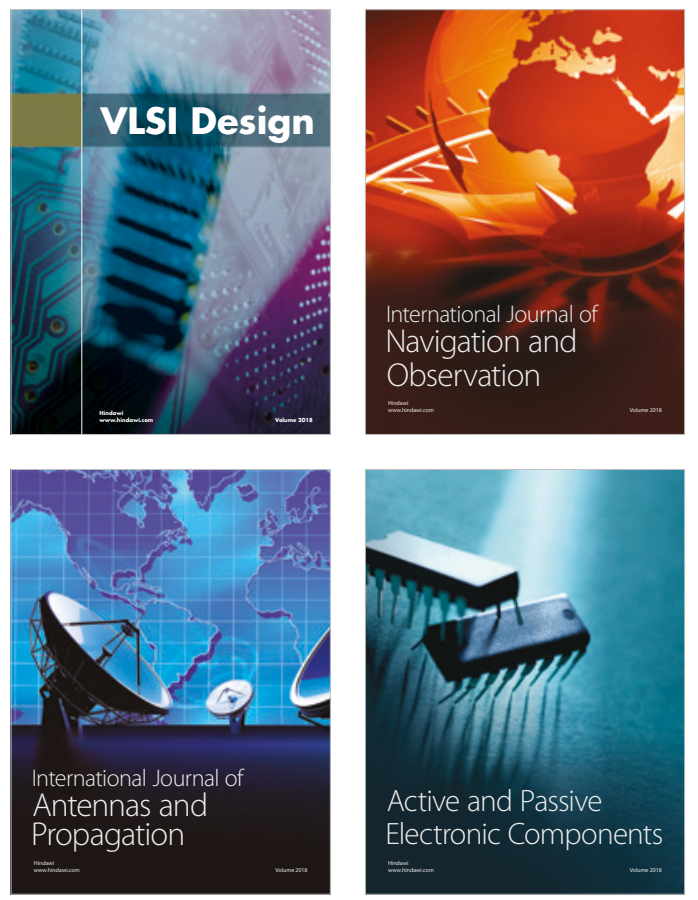
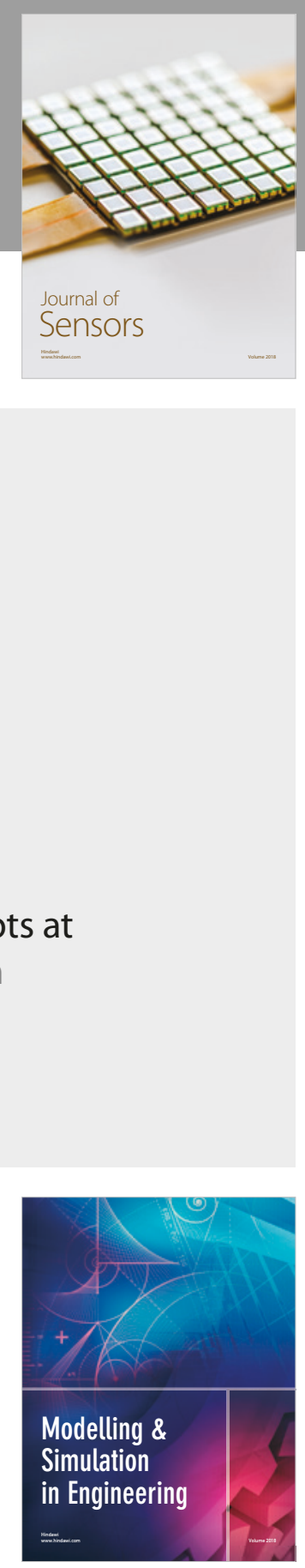

\section{Advances \\ Multimedia}
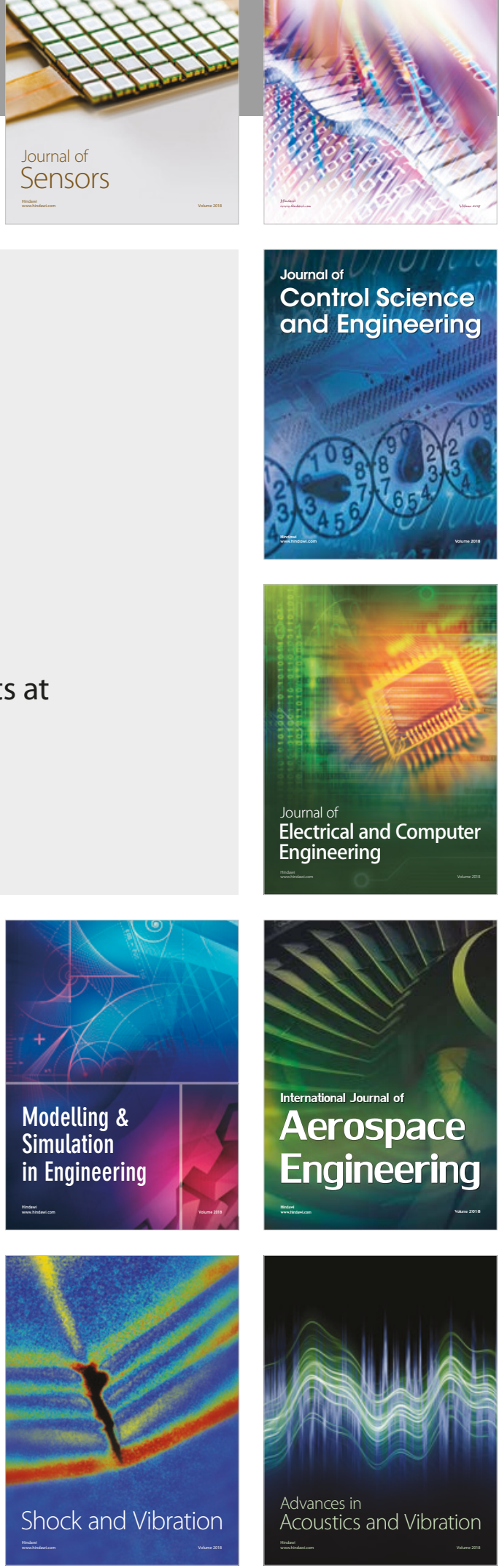